\title{
Changes of EGFR and SMC4 expressions in triple-negative breast cancer and their early diagnostic value
}

\author{
Ting Huang ${ }^{1 \#}$, Jing Xiang ${ }^{2 \#}$, Yun Wang ${ }^{3}$, Youlin Tuo ${ }^{1}$ \\ ${ }^{1}$ Department of Breast Surgery, Sichuan Academy of Medical Sciences \& Sichuan Provincial People's Hospital, School of Medicine, University \\ of Electronic Science \& Technology of China, Chengdu, China; ${ }^{2}$ Department of Outpatient, Sichuan Academy of Medical Sciences \& Sichuan \\ Provincial People's Hospital, School of Medicine, University of Electronic Science \& Technology of China, Chengdu, China; ${ }^{3}$ Department of \\ Neurology, Sichuan Academy of Medical Sciences \& Sichuan Provincial People's Hospital, School of Medicine, University of Electronic Science \& \\ Technology of China, Chengdu, China \\ Contributions: (I) Conception and design: T Huang, J Xiang; (II) Administrative support: Y Tuo; (III) Provision of study materials or patients: T \\ Huang, J Xiang, Y Tuo; (IV) Collection and assembly of data: All authors; (V) Data analysis and interpretation: J Xiang, Y Wang; (VI) Manuscript \\ writing: All authors; (VII) Final approval of manuscript: All authors. \\ \#These authors contributed equally to this work and are co-first authors. \\ Correspondence to: Youlin Tuo. Department of Breast Surgery, Sichuan Academy of Medical Sciences \& Sichuan Provincial People's Hospital, School \\ of Medicine, University of Electronic Science \& Technology of China, No. 32, West Section 2, 1st Ring Road, Qingyang District, Chengdu, China. \\ Email: 157722@163.com; Yun Wang. Department of Neurology, Sichuan Academy of Medical Sciences \& Sichuan Provincial People's Hospital, \\ School of Medicine, University of Electronic Science \& Technology of China, No. 32, West Section 2, 1st Ring Road, Qingyang District, Chengdu, \\ China. Email: lizzywon77@163.com.
}

Background: To explore the diagnostic value of epidermal growth factor receptor (EGFR) and structural maintenance of chromosome protein 4 (SMC4) for triple-negative breast cancer.

Methods: A total of 213 breast cancer patients were selected and divided into triple-negative breast cancer (100 cases) and non-triple-negative breast cancer (113 cases) according to the expression of estrogen receptor (ER), progesterone receptor (PR), and human epidermal growth factor receptor 2 (HER2). Patient information including age, body mass index (BMI), smoking history, drinking history, menopause, tumor classification, lymph node metastasis, distant metastasis, clinical stage, and EGFR and SMC4 expression were collected for all subjects. Logistic regression analysis was then used to evaluate the risk factors for triplenegative breast cancer. The ROC curve was also used to evaluate the clinical value of EGFR and SMC4 in the diagnosis of triple-negative breast cancer.

Results: Logistic regression analysis showed that high expression of SMC4 and high expression of EGFR were both risk factors for triple-negative breast cancer, with an odds ratio (OR) of 1.72 and 1.56, respectively (both $\mathrm{P}<0.05$ ). ROC curve analysis results showed that the areas under the curve with high SMC4 expression and high EGFR expression for the diagnosis of triple-negative breast cancer were 0.84 and 0.78 , respectively. Conclusions: High expression of SMC4 and EGFR is significantly correlated with triple-negative breast cancer, and can be used as an auxiliary diagnostic indicator for triple-negative breast cancer.

Keywords: Triple-negative breast cancer; structural maintenance of chromosome protein 4 (SMC4); epidermal growth factor receptor (EGFR)

Submitted Jan 27, 2021. Accepted for publication Mar 22, 2021.

doi: $10.21037 /$ gs-21-119

View this article at: http://dx.doi.org/10.21037/gs-21-119 


\section{Introduction}

Breast cancer is the most common type of cancer in women. The latest data show that approximately 2.08 million patients are diagnosed with breast cancer every year, and as many as 626,000 patients die of breast cancer every year $(1,2)$. Breast cancer can be divided into triple-negative breast cancer and non-triple-negative breast cancer according to the expression status of 3 receptors: estrogen receptor (ER), progesterone receptor (PR), and human epidermal growth factor receptor 2 (HER2) (3). The clinical features of triplenegative breast cancer include early-onset, a high degree of malignancy, and poor prognosis $(4,5)$.

Structural maintenance of chromosome protein 4 (SMC4) is involved in maintaining the stability of chromosome structure and the mitosis of eukaryotic cells (5). A high expression level of SMC4 is often associated with the malignant proliferation and expansion of tumor cells (6). Studies have also found that a high expression level of SMC4 is related to the reduced survival rate of patients with colorectal cancer (7). Epidermal growth factor receptor (EGFR) is involved in regulating cell differentiation and proliferation (8). Studies have shown that EGFR is frequently overexpressed in lung cancer, liver cancer, prostate cancer, and other cancers, suggesting its clinical significance (9-11). The occurrence and development of breast cancer are closely related to age and the level of sex hormones in the body $(3,4)$. Previous studies have explored the diagnostic value of EGFR and SMC4 for breast cancer in elderly women (over 60 years of age) (11). However, the impact of whether young women and patients are menopausal is not fully considered. This study aims to explore the diagnostic value of EGFR and SMC4 for triplenegative breast cancer in middle-aged and elderly female patients. We present the following article in accordance with the STARD reporting checklist (available at http:// dx.doi.org/10.21037/gs-21-119).

\section{Methods}

\section{Research subjects}

A total of 213 breast cancer patients who were hospitalized in our hospital from January 2016 to January 2020 were selected. Inclusion criteria: (I) female patients who were hospitalized in our hospital for breast cancer surgery from January 2016 to January 2020; (II) all cases had clear pathology (ER, PR, HER2) results and immunohistochemistry results from our hospital. The study conformed to the provisions of the Declaration of Helsinki (as revised in 2013). The study was approved by the ethics committee of Sichuan Academy of Medical Sciences \& Sichuan Provincial People's Hospital (No. ChiECRCT20190184), and all research subjects voluntarily signed an informed consent form.

\section{Study methods}

Clinical information including clinical data, laboratory examination results, pathological examination results, immunohistochemical results, age, body mass index (BMI), smoking history, drinking history, menopause, tumor classification, lymph node metastasis, distant metastasis, clinical stage, and ER, PR, and HER2 status, as well as EGFR and SMC4 mRNA expression were collected for all the patients.

The tumor tissues obtained during surgery were frozen in liquid nitrogen, and the expression of SMC4 in tumor tissues and cells was determined by quantitative real-time PCR. According to The Cancer Genome Atlas (TCGA) database, SMC4 expression level above the median was designated as high SMC4 expression, and SMC4 expression level below the median was designated as low SMC4 expression.

EGFR expression levels were determined from paraffinembedded cancer tissue sections. Specifically, if the staining intensity score of cellular EGFR in the tissue times the number of positive cells was $\geq 3$ points, the sample was judged as EGFR overexpression, and was then recorded as EGFR (+). On the other hand, if the staining intensity score of EGFR times the number of positive cells was $\leq 2$ points, the sample was judged as non-EGFR overexpression, and was then recorded as EGFR (-), as shown in Figure 1.

\section{Statistical analysis}

Normally distributed data were expressed as mean \pm standard deviation, and the difference between the 2 groups was determined using a $t$-test. The $\chi^{2}$ test was used to compare count data. Logistic regression analysis was used to evaluate the risk factors of triple-negative breast cancer. A receiver operating characteristic (ROC) curve was used to evaluate the value of EGFR and SMC4 in the diagnosis of triple-negative breast cancer. $\mathrm{P}<0.05$ was considered statistically significant. All statistical analyses were performed with SPSS 22.0. 

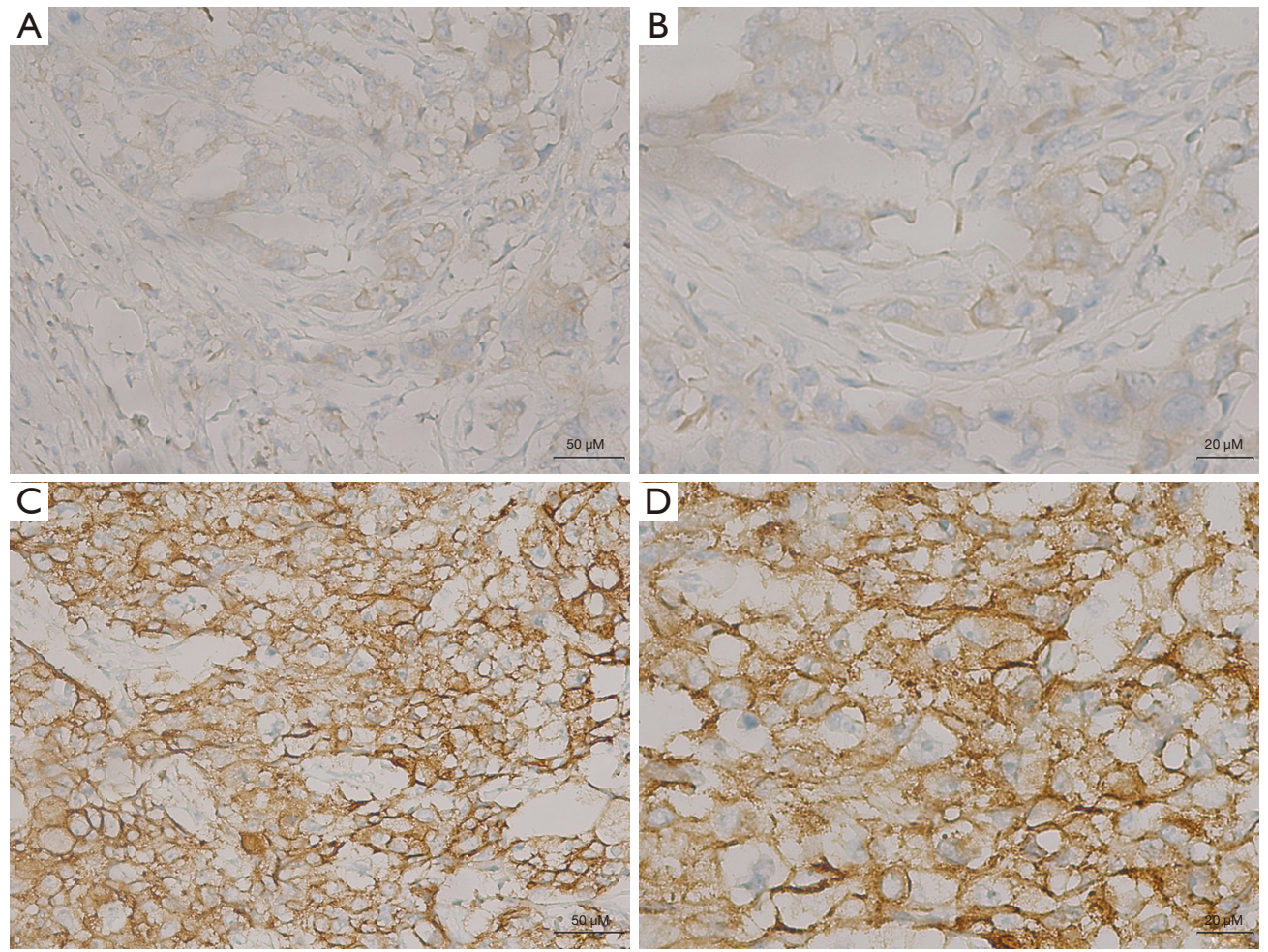

Figure 1 Stain detection of EGFR in triple-negative breast cancer tissues. (A,B) EGFR negative (the number of positive cells was less than or equal to 2 points. A: 20x; B: 40x). (C,D) EGFR positive (the number of positive cells was more than 3 points. C: 20×; D: 40x). EGFR, epidermal growth factor receptor.

\section{Results}

\section{Comparison of various indicators between patients with triple-negative breast cancer and non-triple-negative breast cancer}

The proportions of patients with menopause, breast cancer (T2-T4), clinical stages ranging from I-II, high SMC4 expression, and high EGFR expression in the triple-negative breast cancer group were significantly higher than those in the non-triple-negative breast cancer group (all $\mathrm{P}<0.05)$. The proportions of patients with breast cancer (T1), clinical stages III-IV, low SMC4 expression, and low EGFR expression were significantly lower in the triple-negative breast cancer group than those in the non-triple-negative breast cancer group (all $\mathrm{P}<0.05$ ). There were no significant differences in age, BMI, smoking rates, and alcohol consumption rates between the 2 groups (all $\mathrm{P}>0.05$ ), as shown in Table 1 .

\section{Analysis of risk factors for triple-negative breast cancer}

Among the breast cancer patients with high SMC4 expression, triple-negative breast cancer patients accounted for $65.4 \%$, and among the breast cancer patients with high EGFR expression, triple-negative breast cancer patients accounted for $62.3 \%$. Logistic regression analysis showed that high expression of SMC4 and high expression of EGFR were both risk factors for triple-negative breast cancer with an odds ratio $(\mathrm{OR})$ of 1.72 and 1.56 , respectively, $(\mathrm{P}<0.05)$ as shown in Table 2.

\section{Analysis of the diagnostic efficacy of SMC4 and EGFR for triple-negative breast cancer}

ROC curve analysis was performed for all patients. The results showed that the areas under the curve with SMC4 high expression and EGFR high expression as variables were 0.84 and 0.78 , respectively. Logistic regression analysis was then used to assess the diagnostic efficacy with combined high SMC4 expression and high EGFR expression as the predictor. The AUC of the combined detection model for the diagnosis of triple-negative breast cancer was 0.92 , which was better than individual predictors 
Table 1 Comparison of various indicators between patients with triple-negative breast cancer and non-triple-negative breast cancer

\begin{tabular}{|c|c|c|c|}
\hline Variable & Non-triple-negative breast cancer $(\mathrm{N}=113)$ & Triple-negative breast cancer $(\mathrm{N}=100)$ & $P$ value \\
\hline $\mathrm{BMI}\left(\mathrm{kg} / \mathrm{m}^{2}\right)$ & $24.9 \pm 4.2$ & $25.3 \pm 2.7$ & 0.221 \\
\hline Menopause (n, \%) & $61[53.4]$ & $52[52]$ & 0.004 \\
\hline Smoking (n, \%) & $17[15.0]$ & $10[10]$ & 0.07 \\
\hline \multicolumn{4}{|l|}{ Tumor classification (n, \%) } \\
\hline T1 & 67 [59.3] & $43[43]$ & 0.012 \\
\hline T2-T4 & $46[40.7]$ & $57[57]$ & 0.037 \\
\hline Lymph node metastasis (n, \%) & $21[18.6]$ & $17[17]$ & 0.091 \\
\hline \multicolumn{4}{|l|}{ Clinical stage } \\
\hline$I-I I$ & 82 [72.6] & 90 [90] & 0.002 \\
\hline III-IV & $31[27.4]$ & $10[10]$ & 0.004 \\
\hline \multicolumn{4}{|l|}{ SMC4 expression (n, \%) } \\
\hline Low expression & $77[68.1]$ & 32 [32] & 0.007 \\
\hline High expression & 36 [31.9] & 68 [68] & 0.006 \\
\hline \multicolumn{4}{|l|}{ EGFR expression (n, \%) } \\
\hline Low expression & $69[61.1]$ & 27 [27] & $<0.0001$ \\
\hline High expression & 44 [38.9] & 73 [73] & $<0.0001$ \\
\hline
\end{tabular}

BMI, body mass index; SMC4, structural maintenance of chromosome protein 4; EGFR, epidermal growth factor receptor.

Table 2 Correlation analysis between SMC4, EGFR, and triple-negative breast cancer risk

\begin{tabular}{lccc}
\hline Variable & OR value & $95 \%$ Cl & P value \\
\hline SMC4 high vs. SMC4 low & 1.72 & $1.23-1.93$ & 0.0004 \\
EGFR high vs. EGFR low & 1.56 & $1.34-1.87$ & $<0.0001$ \\
\hline
\end{tabular}

SMC4, structural maintenance of chromosome protein 4; EGFR, epidermal growth factor receptor.

as shown in Table 3 and Figure 2.

\section{Discussion}

Breast cancer is the most common malignant tumor in women. Although the mortality rate of breast cancer has reportedly decreased since 1989 , this decreasing trend has recently slowed down (12). Therefore, new prevention and treatment strategies for breast cancer are in urgent need of research and development.

SMC4 is highly expressed in a variety of cancers, including glioma, colorectal cancer, and hepatocellular carcinoma (13-15). Sq25 is the gene locus of SMC4, which showed a high level of DNA amplification for relapsed breast cancer cell lines (16). Consistent with our findings, SMC4 expression is up-regulated in patients with triplenegative breast cancer. Up-regulated SMC4 can increase the sensitivity of Cdk1 to drive the densifications of chromosomes during mitosis and increase the proliferation and dedifferentiation of cancer cells (17). This explains the higher expression of SMC4 in triple-negative breast cancer with higher malignancy. At the same time, the high 
Table 3 Analysis of the diagnostic efficacy of SMC4 and EGFR for triple-negative breast cancer

\begin{tabular}{lccc}
\hline Variable & AUC $(95 \% \mathrm{Cl})$ & Sensitivity/\% & Specificity/\% \\
\hline SMC4 high & $0.84(0.57-0.91)$ & 85.13 & 47.22 \\
EGFR high & $0.78(0.49-0.82)$ & 75.06 & 82.77 \\
Combined model & $0.92(0.77-0.96)$ & 86.51 & 83.29 \\
\hline
\end{tabular}

SMC4, structural maintenance of chromosome protein 4; EGFR, epidermal growth factor receptor.

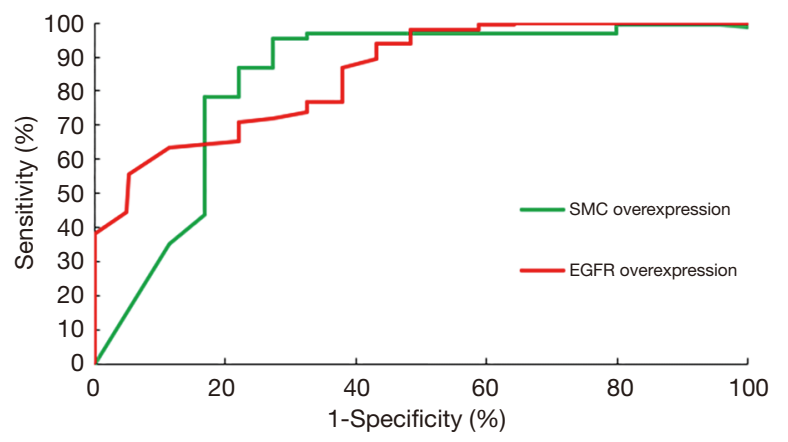

Figure 2 ROC curve of high SMC4 and EGFR expression for the diagnosis of triple-negative breast cancer. ROC, receiver operating characteristic; SMC4, chromosome protein 4; EGFR, epidermal growth factor receptor.

expression of SMC4 may increase double-stranded DNA breaks by enhancing the effect of topoisomerase II $(18,19)$ which leads to mutations and mismatches, resulting in chromosomal rearrangements in breast epithelial cells (20). Studies have confirmed that overexpression of SMC4 can activate the JAK2/STAT3 and TGF $3 /$ Smad signaling pathways, and promotes the invasiveness of cancer cells (21). Previous studies have shown that in ER-positive breast cancer, PLK1 can increase endoplasmic reticulum transcription activity and associated cell invasion $(15,16)$. It has also been shown that the high expression of SMC4 can promote the up-regulation of PLK1 (17). These findings may suggest that SMC4 has a certain correlation with the progression and prognosis of breast cancer, and SMC4 may become an independent predictive prognostic factor and therapeutic target. Studies have also suggested that the potential mechanism of SMC4-associated cell invasiveness may be derived from the PI3K/AKT signaling pathway, and the expression level of SMC4 can affect the activation of this pathway (18). The PI3K/AKT signaling pathway plays an important role in tumor cell proliferation, migration, invasion, and other aspects (19). The activation of PI3K can lead to the production of phosphatidylinositol 3-phosphate (PIP3) in the plasma membrane, which combined with AKT can activate PIP3 catalytic activity and thus participate in the regulation of cell growth, proliferation, and apoptosis (20). When the expression of SMC4 is down-regulated, the activation of the PI3K/AKT signaling pathway may be deactivated, and the proliferation, migration, and invasion of breast cancer cells may therefore be inhibited.

It has been shown that during the occurrence and development of cancer, the signal transduction pathway mediated by EGFR plays an important role (22). An increasing number of studies have shown that EGFR is overexpressed in a variety of human malignant tumor tissues (23). Cohort studies have also found that patients with EGFR overexpression in tumor tissues tend to have a shorter tumor recurrence time, a higher recurrence rate, and a shorter survival period (24). Therefore, the specific high expression of EGFR in malignant tumors provides opportunities for early diagnosis and prevention of triple-negative breast cancer. Similar to a previously report by Bhola et al. in which the high expression rate of EGFR was (46-71\%) in triplenegative breast cancer, the high expression rate of EGFR in triple-negative breast cancer patients in this study was $73 \%$, which was also verified by its effective diagnostic values for triple-negative breast cancer. The underlying mechanism may be that the EGFR signal transduction pathway induces cell malignancy, which mediates the proliferation and invasion of tumor cells and the growth and expansion of tumor-associated blood vessels (22). Furthermore, it has been suggested that with the progression of triple-negative breast cancer, the mediating role of EGFR is continuously strengthened by positive feedback, thus accelerating the progression of the disease (23).

In summary, the expression levels of SMC4 and EGFR in patients with triple-negative breast cancer and those with non-triple-negative breast cancer are significantly different. High expression levels of SMC4 and EGFR are risk factors for the occurrence of triple-negative breast cancer. Furthermore, the combined detection of SMC4 and 
EGFR can improve the accuracy of diagnosis and provide important supporting evidence for the early diagnosis of triple-negative breast cancer.

\section{Acknowledgments}

Funding: None.

\section{Footnote}

Reporting Checklist: The authors have completed the STARD reporting checklist. Available at http://dx.doi.org/10.21037/ gs-21-119

Data Sharing Statement: Available at http://dx.doi. org/10.21037/gs-21-119

Conflicts of Interest: All authors have completed the ICMJE uniform disclosure form (available at http://dx.doi. org/10.21037/gs-21-119). The authors have no conflicts of interest to declare.

Ethical Statement: The authors are accountable for all aspects of the work in ensuring that questions related to the accuracy or integrity of any part of the work are appropriately investigated and resolved. The study conformed to the provisions of the Declaration of Helsinki (as revised in 2013). The study was approved by the ethics committee of Sichuan Academy of Medical Sciences \& Sichuan Provincial People's Hospital (No. ChiECRCT20190184), and all research subjects voluntarily signed an informed consent form.

Open Access Statement: This is an Open Access article distributed in accordance with the Creative Commons Attribution-NonCommercial-NoDerivs 4.0 International License (CC BY-NC-ND 4.0), which permits the noncommercial replication and distribution of the article with the strict proviso that no changes or edits are made and the original work is properly cited (including links to both the formal publication through the relevant DOI and the license). See: https://creativecommons.org/licenses/by-nc-nd/4.0/.

\section{References}

1. Bray F, Ferlay J, Soerjomataram I, et al. Global cancer statistics 2018: GLOBOCAN estimates of incidence and mortality worldwide for 36 cancers in 185 countries. CA
Cancer J Clin 2018;68:394-424.

2. Chen $W$, Zheng R, Baade PD, et al. Cancer statistics in China, 2015. CA Cancer J Clin 2016;66:115-32.

3. Kumar P, Aggarwal R. An overview of triple-negative breast cancer. Arch Gynecol Obstet 2016;293:247-69.

4. Yin L, Duan JJ, Bian XW, et al. Triple-negative breast cancer molecular subtyping and treatment progress. Breast Cancer Res 2020;22:61.

5. Lyons TG. Targeted Therapies for Triple-Negative Breast Cancer. Curr Treat Options Oncol 2019;20:82.

6. Zhang C, Kuang M, Li M, et al. SMC4, which is essentially involved in lung development, is associated with lung adenocarcinoma progression. Sci Rep 2016;6:34508.

7. Nishiwaki T, Daigo Y, Kawasoe T, et al. Isolation and characterization of a human cDNA homologous to the Xenopus laevis XCAP-C gene belonging to the structural maintenance of chromosomes (SMC) family. J Hum Genet 1999;44:197-202.

8. Fraguas $\mathrm{S}$, Barberán S, Cebrià F. EGFR signaling regulates cell proliferation, differentiation and morphogenesis during planarian regeneration and homeostasis. Dev Biol 2011;354:87-101.

9. Leonetti A, Sharma S, Minari R, et al. Resistance mechanisms to osimertinib in EGFR-mutated non-small cell lung cancer. Br J Cancer 2019;121:725-37.

10. Fu J, Wang $\mathrm{H}$. Precision diagnosis and treatment of liver cancer in China. Cancer Lett 2018;412:283-8.

11. Ma RM, Yang F, Huang DP, et al. The Prognostic Value of the Expression of SMC4 mRNA in Breast Cancer. Dis Markers 2019 Nov 21;2019:2183057.

12. Fahad Ullah M. Breast Cancer: Current Perspectives on the Disease Status. Adv Exp Med Biol 2019;1152:51-64.

13. Takahashi S, Fusaki N, Ohta S, et al. Downregulation of KIF23 suppresses glioma proliferation. J Neurooncol 2012;106:519-29.

14. Li X, Chen W, Jia J, et al. The Long Non-Coding RNARoR Promotes the Tumorigenesis of Human Colorectal Cancer by Targeting miR-6833-3p Through SMC4. Onco Targets Ther 2020;13:2573-81.

15. Zhou B, Chen H, Wei D, et al. A novel miR-219-SMC4JAK2/Stat3 regulatory pathway in human hepatocellular carcinoma. J Exp Clin Cancer Res 2014;33:55.

16. Forozan F, Mahlamäki EH, Monni O, et al. Comparative genomic hybridization analysis of 38 breast cancer cell lines: a basis for interpreting complementary DNA microarray data. Cancer Res 2000;60:4519-25.

17. Robellet $X$, Thattikota $Y$, Wang F, et al. A high-sensitivity phospho-switch triggered by Cdk1 governs chromosome 
morphogenesis during cell division. Genes Dev 2015;29:426-39.

18. Feng XD, Song Q, Li CW, et al. Structural maintenance of chromosomes 4 is a predictor of survival and a novel therapeutic target in colorectal cancer. Asian Pac J Cancer Prev 2014;15:9459-65.

19. Jiang L, Zhou J, Zhong D, et al. Overexpression of SMC4 activates TGF $\beta /$ Smad signaling and promotes aggressive phenotype in glioma cells. Oncogenesis 2017;6:e301.

20. Kulawiec M, Safina A, Desouki MM, et al. Tumorigenic transformation of human breast epithelial cells induced by mitochondrial DNA depletion. Cancer Biol Ther 2008;7:1732-43.

21. Kang GY, Lee ER, Kim JH, et al. Downregulation of

Cite this article as: Huang T, Xiang J, Wang Y, Tuo Y. Changes of EGFR and SMC4 expressions in triple-negative breast cancer and their early diagnostic value. Gland Surg 2021;10(3):1118-1124. doi: 10.21037/gs-21-119
PLK-1 expression in kaempferol-induced apoptosis of MCF-7 cells. Eur J Pharmacol 2009;611:17-21.

22. Roskoski R Jr. Small molecule inhibitors targeting the EGFR/ErbB family of protein-tyrosine kinases in human cancers. Pharmacol Res 2019;139:395-411.

23. Tomas A, Futter CE, Eden ER. EGF receptor trafficking: consequences for signaling and cancer. Trends Cell Biol 2014;24:26-34.

24. Yao YD, Sun TM, Huang SY, et al. Targeted delivery of PLK1-siRNA by ScFv suppresses Her2+ breast cancer growth and metastasis. Sci Transl Med 2012;4:130ra48.

(English Language Editor: C. Betlazar-Maseh) 